\title{
Erratum: Self-consistent time dependent two dimensional and three dimensional space charge distributions with linear force [Phys. Rev. ST Accel. Beams 6, 094202 (2003)]
}

\author{
V. Danilov, S. Cousineau, S. Henderson, and J. Holmes
}

(Received 7 January 2008; published 16 January 2008)

We have detected some typographical errors in our paper on self-consistent 2D and 3D space charge distributions. The first error appears in Eq. (37): the argument of the tangent should be $2 \varphi$, rather than $\varphi$. The equation should read:

$$
\operatorname{tg} 2 \varphi=-\frac{2(f b+e a)}{a^{2}+b^{2}-e^{2}-f^{2}} .
$$

The second error is that the coefficients $c_{1}$ and $c_{2}$ in Eq. (38)

$$
\frac{x_{N}^{2}}{c_{1}^{2}}+\frac{y_{N}^{2}}{c_{2}^{2}}=1 \text {, }
$$

miss a factor of $(a f-e b)$. The equations for the coefficients should read $c_{1}^{2}=(a f-e b)^{2} /\left[\left(e^{2}+f^{2}\right) \cos ^{2} \varphi+\left(a^{2}+\right.\right.$ $\left.\left.b^{2}\right) \sin ^{2} \varphi+2(f b+e a) \cos \varphi \sin \varphi\right], c_{2}^{2}=(a f-e b)^{2} /\left[\left(e^{2}+f^{2}\right) \sin ^{2} \varphi+\left(a^{2}+b^{2}\right) \cos ^{2} \varphi-2(f b+e a) \cos \varphi \sin \varphi\right]$.

The third error appears in Eqs. (41) for the constants $c, d, g, h$ have differentiation on the wrong side of the equations. They should read:

$$
c=a^{\prime}, \quad d=b^{\prime}, \quad g=e^{\prime}, \quad h=f^{\prime} .
$$

We apologize for the mistakes, but note that these equations were presented as an example, were never used in the paper, and the paper's results remain unchanged. 